Pacific Journal of Mathematics

ASYMPTOTICS FOR SOLUTIONS OF SYSTEMS OF SMOOTH 


\title{
ASYMPTOTICS FOR SOLUTIONS OF SYSTEMS OF SMOOTH RECURRENCE EQUATIONS
}

\author{
William C. Bauldry, Attila Máté and Paul Nevai
}

It is shown that convergent solutions of a system of smooth recurrence equations whose Jacobian matrix satisfies a certain "nonunimodularity" condition can be approximated by asymptotic expansions. An application is given to approximate the recurrence coefficients associated with polynomials orthonormal with respect to the weight $\exp (-Q(x))$, where $Q$ is an even degree polynomial with positive leading coefficients.

1. Introduction and the main results. The aim of these notes is to generalize the results of [9] to systems of recurrence equations. As will be discussed in $\S \S 4$ and 5 , the need for such a generalization arose in connection with systems of recurrence equations describing certain coefficients connected with orthogonal polynomials associated with asymmetric Freud weights on the real line. Our main result is

THEOREM 1.1. Let $k \geq 0, N \geq 1$, and $r \geq 1$ be integers, and for each integer $\mu$ with $1 \leq \mu \leq r$ let

$$
H_{\mu}\left(x_{0} ; x_{\nu j}: 1 \leq \nu \leq r, 0 \leq j \leq k\right)
$$

be a complex-valued function of $(k+1) r+1$ real variables $x_{0}, x_{\nu j}$, all of whose partial derivatives of order $\leq N$ are continuous in a neighborhood of the origin 0 . Denoting by $\partial_{0}$ and $\partial_{\nu j}$ the partial derivatives $\partial / \partial x_{0}$ and $\partial / \partial x_{v j}$, respectively, assume that

$$
\operatorname{det}\left[\sum_{j=0}^{k} z^{j} \partial_{\nu j} H_{\mu}(0)\right]_{1 \leq \mu, \nu \leq r} \neq 0
$$

holds for all complex numbers $z$ with $|z|=1$ (det indicates the determinant of the $r \times r$ matrix on the left).

Let $\beta<0$ be a fixed real number, and assume that the reals $y_{\nu n}$ $(1 \leq \nu \leq r, n \geq 1)$ with

$$
\lim _{n \rightarrow \infty} y_{\nu n}=0 \quad(1 \leq \nu \leq r)
$$

form a solution of the system

$$
H_{\mu}\left(n^{\beta} ; y_{\nu, n+j}: 1 \leq \nu \leq r, 0 \leq j \leq k\right)=0 \quad(1 \leq \mu \leq r, n \geq 1)
$$


of recurrence equations. Write

$$
S=\{\beta i-j: i>0, j \geq 0, \beta i-j \geq \beta N\},
$$

and let $\beta=s_{1}>s_{2}>\cdots>s_{q}=\beta N$ be an enumeration of the elements of the set $S$. Then there are numbers $c_{\nu l}(1 \leq \nu \leq r, 1 \leq l \leq q)$ such that

$$
y_{\nu n}=\sum_{l=1}^{q} c_{\nu l} n^{s_{l}}+o\left(n^{\beta N}\right) \text { as } n \rightarrow \infty \text {. }
$$

Moreover, the numbers $c_{\nu l}$ depend only on the ith partial derivatives of $H_{\mu}$ for $1 \leq \mu \leq r$ and $1 \leq i \leq N$.

If $1 / \beta$ is an integer then clearly

$$
S=\{\beta l: 1 \leq l \leq N\},
$$

and so (1.5) simplifies to

$$
y_{\nu n}=\sum_{l=1}^{N} c_{\nu l} n^{\beta l}+o\left(n^{\beta N}\right) .
$$

The proof of this theorem depends on the following result about systems of linear recurrence equations; this result generalizes the Lemma of $[9$, p. 424]:

LEMMA 1.2. Let $k \geq 0$ and $r \geq 1$ be integers, and let $f_{\nu}$ and $g_{\mu}$ $(1 \leq \mu \leq r, 1 \leq \nu \leq r)$ be complex-valued functions defined on positive integers such that the equations

$$
\sum_{\nu=1}^{r} \sum_{j=0}^{k} \lambda_{\mu \nu n j} f_{\nu}(n+j)=g_{\mu}(n) \quad(n>0,1 \leq \mu \leq r)
$$

hold, where $\lambda_{\mu \nu n j}$ are complex numbers such that the limits

$$
\lim _{n \rightarrow \infty} \lambda_{\mu \nu n j}=\lambda_{\mu \nu j} \quad(1 \leq \mu, \nu \leq r ; \quad 0 \leq j \leq k)
$$

exist. Suppose that the "characteristic determinant"

$$
D(z)=\operatorname{det}\left[\sum_{j=0}^{k} \lambda_{\mu \nu j} z^{j}\right]_{1 \leq \mu, \nu \leq r}
$$

satisfies

$$
D(z) \neq 0
$$

for every complex $z$ with $|z|=1$. 
Now let $\alpha<0$ be a fixed real number, and suppose that each $f_{\nu}$ is bounded $(1 \leq \nu \leq r)$, and

$$
\lim _{n \rightarrow \infty} g_{\mu}(n) n^{-\alpha}=0 \quad(1 \leq \mu \leq r) .
$$

Then we have

$$
\lim _{n \rightarrow \infty} f_{\nu}(n) n^{-\alpha}=0 \quad(1 \leq \nu \leq r) .
$$

as well.

Next we turn to the proofs of these results. The proofs are closely related to those in [9], though the present results are substantially more general. A special case of Theorem 1.1 was given in [1] without proof.

2. Proof of Lemma 1.2. In virtue of $(1.10), 1 / D(z)$ has a Laurent expansion

$$
1 / D(z)=\sum_{l=-\infty}^{\infty} a_{l} z^{l}
$$

absolutely convergent in a closed annulus $\rho^{-1} \leq|z| \leq \rho(\rho>1)$. This means in particular that

$$
a_{l}=o\left(\rho^{-|l|}\right) \quad \text { as } l \rightarrow \pm \infty .
$$

Put $\lambda_{\mu \nu n j}=\lambda_{\mu \nu j}$ for $n \leq 0$, and extend $f_{\nu}$ and $g_{\mu}$ to arguments $n \leq 0$ as follows $(1 \leq \mu, \nu \leq r ; \quad 0 \leq j \leq k)$. Put $f_{\nu}(n)=0$ for all $n \leq 0$ and then determine $g_{\mu}(n)$ for $n \leq 0$ from (1.7). Clearly, we will have $g_{\mu}(n)=0$ for all but finitely many $n \leq 0$, and (1.7) will be valid for all integers $n$ with $-\infty<n<\infty$. Using $E$ to denote the forward shift operator, that is

$$
E^{l} f(n)=f(n+l) \quad(-\infty<l<\infty),
$$

and writing

$$
h_{\mu}(n)=g_{\mu}(n)+\sum_{\nu=1}^{r} \sum_{j=0}^{k}\left(\lambda_{\mu \nu j}-\lambda_{\mu \nu n j}\right) f_{\nu}(n+j)
$$

this means that

$$
\sum_{\nu=1}^{r}\left(\sum_{j=0}^{k} \lambda_{\mu \nu j} E^{j}\right) f_{\nu}(n)=h_{\mu}(n) \quad(-\infty<n<\infty, 1 \leq \mu \leq r)
$$

holds. Using Cramer's rule, we can solve this system of equations, except for the division by $D(E)$ (cf. (1.9)), to be discussed later; the point is that (2.4) is a system of linear equations where the coefficients on the left-hand 
side come from a commutative ring. We obtain

$$
D(E) f_{\nu}(n)=\sum_{\mu=1}^{r} D_{\mu \nu}(E) h_{\mu}(n),
$$

where $D_{\mu \nu}(E)$ is the cofactor of the element in the $\mu$ th row and the $\nu$ th column of the determinant $D(E)$. The right-hand side looks like the expansion of the determinant corresponding to the $\nu$ th unknown, but it is not a determinant since $D_{\mu \nu}(E)$ and $h_{\mu}(n)$ do not commute in general. From (2.5) one can obtain $f_{\nu}(n)$ via multiplying both sides by the inverse of $D(E)$. According to $(2.1)$, the inverse of $D(E)$ is formally given by

$$
\sum_{l=-\infty}^{\infty} a_{l} E^{l}
$$

hence, one might surmise that

$$
f_{\nu}(n)=\sum_{l=-\infty}^{\infty} a_{l} E^{l} \sum_{\mu=1}^{r} D_{\mu \nu}(E) h_{\mu}(n) .
$$

To see that this formal calculation is indeed correct, one only has to observe that the series on the right-hand side is absolutely convergent in view of (2.2) and the boundedness of $h_{\mu}$. The boundedness of $h_{\mu}$ follows from (1.8), (1.11), and the assumed boundedness of $f_{\nu}$ for $1 \leq \nu \leq r$ (cf. (2.3)).

(2.6) allows us to estimate $f_{\nu}(n)$ for large positive $n$ as follows. Using (2.3), (2.6) can be rewritten as

$$
\begin{aligned}
f_{\nu}(n)= & \sum_{l=-\infty}^{\infty} \sum_{\mu=1}^{r} a_{l} D_{\mu \nu}(E) g_{\mu}(n+l) \\
& +\sum_{l=-\infty}^{\infty} \sum_{\mu=1}^{r} \sum_{s=1}^{r} \sum_{j=0}^{k} a_{l} D_{\mu \nu}(E)\left(\lambda_{\mu s j}-\lambda_{\mu s j}(n+l)\right) f_{s}(n+l) ;
\end{aligned}
$$

here we used the notation $\lambda_{\mu \nu j}(n)=\lambda_{\mu \nu n j}$ to indicate $n$ as the argument on which the operator $E$ acts; moreover, we incorporated the powers $E^{l}$ into the functions as arguments shifts. By (1.11) and (2.2), the first sum on the right-hand side of (2.7) is $o\left(n^{\alpha}\right)$ as $n \rightarrow \infty$. As for the second sum, the absolute value of the term corresponding to the indices $l, \mu, s, j$ is

$$
\leq K\left|a_{l}\right| \max _{0 \leq i \leq(r-1) k}\left|\lambda_{\mu s j}-\lambda_{\mu s j}(n+l+i)\right|\left|f_{s}(n+l+i)\right|,
$$

where $K$ is a constant depending on the determinants $D_{\mu \nu}(E)(1 \leq$ $\mu, \nu \leq r)$, and the range $0 \leq i \leq(r-1) k$ is explained by the fact that the 
highest power of $E$ occurring in (the expansion of) $D_{\mu \nu}(E)$ has exponent at most $(r-1) k$, and so $D_{\mu \nu}(E)$ causes various argument shifts by numbers $i$ in the range described. As the quantity following the symbol max is bounded in view of (1.8) and the boundedness of $f_{s}$, the sum of these terms for $\mu, s, j$, and for $l$ in the range $-\infty<l \leq-n / 2$ is

$$
o\left(\sum_{l=-\infty}^{-n / 2} \rho^{-|l|}\right)=o\left(n^{\alpha}\right) \quad \text { as } n \rightarrow \infty,
$$

according to (2.2). By (1.8), the maximum in (2.8) is

$$
o\left(\sup _{i \geq n+l}\left|f_{s}(i)\right|\right) \text { as } n+l \rightarrow \infty \text {. }
$$

Hence the sum of the terms for $\mu, s, j$ and for $l$ in the range $-n / 2<l<\infty$ is

$$
o\left(\sup _{i \geq n / 2} \sum_{s=1}^{r}\left|f_{s}(i)\right|\right)
$$

in view of (2.2).

Putting these estimates together, (2.7) implies that for every $\varepsilon>0$ there is an $n_{\varepsilon}$ such that

$$
\left|f_{\nu}(n)\right| \leq \frac{\varepsilon}{r} n^{\alpha}+\frac{\varepsilon}{r} \sup _{i \geq n / 2} \sum_{s=1}^{r}\left|f_{s}(i)\right|
$$

holds for $n \geq n_{\varepsilon}(>0)$ and for $1 \leq \nu \leq r$. As $\alpha<0, n^{\alpha}$ here is a decreasing function. Thus, putting

$$
F(x)=\sup _{i \geq x} \sum_{s=1}^{r}\left|f_{s}(i)\right|
$$

for any real $x$ ( $F$ is finite since $f_{s}$ is bounded), this means that

$$
F(x) \leq \varepsilon x^{\alpha}+\varepsilon F(x / 2)
$$

holds for every $x \geq n_{\varepsilon}$. Using this repeatedly, with $x / 2^{l}$ replacing $x$ for $0 \leq l \leq q$, where $q$ is the largest integer $\leq \log _{2}\left(x / n_{\varepsilon}\right)$, we obtain that

$$
F(x) \leq \sum_{l=0}^{\infty} \varepsilon^{l+1}\left(\frac{x}{2^{l}}\right)^{\alpha}+\varepsilon^{q+1} F\left(\frac{x}{2^{q+1}}\right) .
$$

Noting that $F\left(x / 2^{q+1}\right) \leq F(0)$ and $\varepsilon^{q+1}=O\left(x^{\log _{2} \varepsilon}\right)$ as $x \rightarrow \infty$ (for fixed $\varepsilon), F(x)=o\left(x^{\alpha}\right)$ follows from here by observing that $\varepsilon>0$ was arbitrary (but $n_{\varepsilon}$ depends on $\varepsilon$ ). Thus (1.12) follows. The proof of Lemma 1.2 is complete. 
3. Proof of Theorem 1.1. Observe that $H_{\mu}(0)=0$ for $1 \leq \mu \leq r$ in view of (1.2) and (1.3). Thus, according to Taylor's formula,

$$
\begin{aligned}
& H_{\mu}\left(n^{\beta} ; y_{\nu, n+j}: 1 \leq \nu \leq r, 0 \leq j \leq k\right) \\
& =\sum_{l=1}^{N-1} \frac{1}{l !}\left(n^{\beta} \partial_{0}+\sum_{\nu=1}^{r} \sum_{j=0}^{k} y_{\nu, n+j} \partial_{\nu j}\right)^{l} H_{\mu}(0) \\
& +\frac{1}{N !}\left(n^{\beta} \partial_{0}+\sum_{\nu=1}^{r} \sum_{j=0}^{k} y_{\nu, n+j} \partial_{\nu j}\right)^{N} H_{\mu}\left(\theta n^{\beta} ; \theta y_{\nu, n+j}: 1 \leq \nu \leq r, 0 \leq j \leq k\right)
\end{aligned}
$$

for $1 \leq r \leq \mu$ and for some $\theta$ (depending on $\mu$ and $n$ ) with $0<\theta<1$, provided $n$ is large enough (so that the point $\left(n^{\beta} ; y_{\nu, n+j}: 1 \leq \nu \leq r\right.$, $0 \leq j \leq k$ ) belongs to a convex neighborhood of 0 in which $H_{\mu}$ is $N$ times continuously differentiable). The left-hand side here is zero according to (1.3). In view of the continuity of the $N$ th derivatives of $H_{\mu}$ at $0,(1.2)$ and the negativity of $\beta$ imply that the right-hand side will change only slightly if we replace the argument of $H$ with 0 in the last term; estimating the magnitude of this change, we obtain the following (note that the modified last term of the preceding formula being incorporated into the sum below, $l$ now goes to $N$ rather than $N-1)$ :

$$
\begin{aligned}
& \sum_{l=1}^{N} \frac{1}{l !}\left(n^{\beta} \partial_{0}+\sum_{\nu=1}^{r} \sum_{j=0}^{k} y_{\nu, n+j} \partial_{\nu j}\right)^{l} H_{\mu}(0) \\
& =o\left(n^{\beta N}\right)+o\left(\sum_{\nu=1}^{r} \sum_{j=0}^{k}\left|y_{\nu, n+j}\right|^{N}\right)
\end{aligned}
$$

as $n \rightarrow \infty$ (the function expressed as $o$ may depedn on $k, r, N$ and the bounds of the $N$ th derivatives of $H_{\mu}$ close to 0 ).

To prove (1.5), we will use induction, that is, we will assume that for some integer $m$ with $1 \leq m \leq q$ we have

$$
y_{\nu n}=\sum_{l=1}^{m-1} c_{\nu l} n^{s_{l}}+\delta_{\nu n} \quad(1 \leq \nu \leq r),
$$

where

$$
\delta_{\nu n}=o\left(n^{s_{m-1}}\right) \text { as } n \rightarrow \infty ;
$$

here in case $m=1$ we put $s_{0}=0$. For $m=1$, (3.3) is simply the restatement of (1.2), and for $m>1$ (3.3) will be the hypothesis of induction. As for the induction step, we will show that expansion (3.2) can 
be continued, i.e. that

$$
\delta_{\nu n}=c_{\nu m} n^{s_{m}}+o\left(n^{s_{m}}\right) .
$$

As we have $s_{q}=\beta N$, in case $m=q$ the error term here will be $o\left(n^{\beta N}\right)$, i.e. the same as in (1.5). Thus, to prove (1.5) it will be sufficient to establish (3.4).

According to (3.2), we have

$$
\begin{aligned}
y_{\nu, n+j} & =\sum_{l=1}^{m-1} c_{\nu l}(n+j)^{s_{l}}+\delta_{\nu, n+j} \\
& =\sum_{l=1}^{m} c_{\nu l j} n^{s_{l}}+\delta_{\nu, n+j}+o\left(n^{s_{m}}\right)
\end{aligned}
$$

as $n \rightarrow \infty$ for, say, $0 \leq j \leq k$. Note that the summation on the right goes to $m$ rather than $m-1$. The right-hand side here is obtained by taking the binomial expansion

$$
(n+j)^{s_{l}}=n^{s_{l}}\left(1+\frac{j}{n}\right)^{s_{l}}=\sum_{s=0}^{\infty}\left(\begin{array}{c}
s_{l} \\
s
\end{array}\right) j^{s n^{s_{l}-s}} .
$$

It is clear from the definition of the set $S$ given in (1.4) that the exponents $s_{l}-s$ of $n$ on the right satisfying $s_{l}-s \geq s_{m}$ belongs to $S$. Thus one indeed obtains the right-hand side of (3.5), and it is clear that the coefficients $c_{\nu l j}(1 \leq l \leq m)$ are determined by the coefficients $c_{\nu l}(1 \leq$ $l \leq m-1)$.

We are going to substitute (3.5) into (3.1). Note for this that $\delta_{\nu, n+j}=$ $o(1)$ and $n^{\beta} \delta_{\nu, n+j}=o\left(n^{s_{m}}\right)$ as $n \rightarrow \infty(1 \leq \nu \leq r, 0 \leq j \leq k)$ according to (3.3); the second relation holds since clearly $s_{m-1}+\beta \leq s_{m}$. As $\beta \geq s_{l}$, the second relation also means that $n^{s_{l}} \delta_{n+j}=o\left(n^{s_{m}}\right)$. Thus carrying out the indicated substitution, we obtain that

$$
\begin{gathered}
\sum_{l=1}^{m-1} C_{\mu l} n^{s_{l}}+C_{\mu m}^{\prime} n^{s_{m}}+\sum_{\nu=1}^{r} \sum_{j=0}^{k} \delta_{\nu, n+j} \delta_{\nu j} \partial_{\nu j} H_{\mu}(0) \\
=o\left(\sum_{\nu=1}^{r} \sum_{j=0}^{k}\left|\delta_{\nu, n+j}\right|\right)+o\left(n^{s_{m}}\right)
\end{gathered}
$$

holds for $1 \leq \mu \leq r$ as $n \rightarrow \infty$ with some constants $C_{\mu l}, 1 \leq l<m$, and $C_{\mu m}^{\prime}$. The first error term on the right comes from the second error term on the right-hand side of (3.1) and from powers higher than first of $\delta_{\nu, n+j}$ resulting from substituting (3.5) into (3.1). Note also that the first error term on the right-hand side of (3.1) was absorbed into the second error term on the right of (3.6); this can be done since $\beta N \leq s_{m}$. It is clear from 
the definition of $S$ in (1.4) that in deriving (3.6), only exponents of $n$ belonging to $S$ will occur, that is no powers of $n$ other than those indicated should occur in (3.6). Substituting (3.3) into (3.6), it follows that $C_{\mu l}=0$ for $1 \leq l \leq m-1$. Thus (3.6) becomes

(3.7) $C_{\mu m}^{\prime} n^{s_{m}}+\sum_{\nu=1}^{r} \sum_{j=0}^{k} \delta_{\nu, n+j} \partial_{\nu j} H_{\mu}(0)=o\left(\sum_{\nu=1}^{r} \sum_{j=0}^{k}\left|\delta_{\nu, n+j}\right|\right)+o\left(n^{s_{m}}\right)$.

As we might have pointed out right after (3.6), the coefficients $C_{\mu m}^{\prime}$ here are determined by the $c_{\nu l}(1 \leq l \leq r, 1 \leq \nu \leq m-1)$ in (3.2) and by the $i$ th order partial derivatives of $H_{\mu}$ at 0 for $1 \leq i \leq N$.

Choose $c_{\nu m}(1 \leq \nu \leq r)$ as the solution of the system

$$
\sum_{\nu=1}^{r} c_{\nu m}\left(\sum_{j=0}^{k} \partial_{\nu j} H_{\mu}(0)\right)=-C_{\mu m}^{\prime} \quad(1 \leq \mu \leq r)
$$

of linear equations. Observe that this system is uniquely solvable according to (1.1) with $z=1$. Put

$$
f_{\nu}(n)=\delta_{\nu, n}-c_{\nu m} n^{s_{m}} \quad(1 \leq \nu \leq r)
$$

In order to establish (3.4), it will be sufficient to show that

$$
f_{\nu}(n)=o\left(n^{s_{m}}\right) \text { as } n \rightarrow \infty \text {. }
$$

To show this, substituting (3.8) and (3.9) into (3.7), we obtain

$$
\begin{gathered}
\sum_{\nu=1}^{r} \sum_{j=0}^{k}\left(f_{\nu}(n+j)+c_{\nu m}\left((n+j)^{s_{m}}-n^{s_{m}}\right)\right) \partial_{\nu j} H_{\mu}(0) \\
=o\left(\sum_{\nu=1}^{r} \sum_{j=0}^{k}\left|\delta_{\nu, n+j}\right|\right)+o\left(n^{s_{m}}\right)
\end{gathered}
$$

for $1 \leq \mu \leq r$ as $n \rightarrow \infty$. The coefficient of $c_{\nu m}$ on the left is $o\left(n^{s_{m}}\right)$, and so we obtain

$$
\sum_{\nu=1}^{r} \sum_{j=0}^{k} \lambda_{\mu \nu j} f_{\nu}(n+j)=\sum_{\nu=1}^{r} \sum_{j=0}^{k} o\left(\left|\delta_{\nu, n+j}\right|\right)+o\left(n^{s_{m}}\right)
$$

where $\lambda_{\mu \nu j}=\partial_{\nu j} H_{\mu}(0)$. Note that with this choice of $\lambda_{\mu \nu j}$, (1.10) is satisfied according to (1.1). By (3.9), for the first error term in (3.11) we have

$$
\begin{aligned}
o\left(\left|\delta_{\nu, n+j}\right|\right) & =o\left(\left|f_{\nu}(n+j)\right|\right)+o\left(n^{s_{m}}\right) \\
& =\eta_{\mu \nu n j} f_{\nu}(n+j)+o\left(n^{s_{m}}\right)
\end{aligned}
$$


with suitable $\eta_{\mu \nu n j}$ such that $\eta_{\mu \nu n j} \rightarrow 0$ as $n \rightarrow \infty$. Thus (3.11) becomes

$$
\sum_{\nu=1}^{r} \sum_{j=0}^{k}\left(\lambda_{\mu \nu j}-\eta_{\mu \nu n j}\right) f_{\nu}(n+j)=o\left(n^{s_{m}}\right)
$$

for $1 \leq \mu \leq r$. Using Lemma 1.2 with $\lambda_{\mu \nu n j}=\lambda_{\mu \nu j}-\eta_{\mu \nu n j}$ for this system of recurrences (note that $f_{\nu}$ is bounded in view of (3.3) and (3.9)), we can conclude that (3.10) is indeed valid. This establishes (3.4), and thus the conclusion of Theorem 1.1 follows by induction. The proof is complete.

4. Asymptotic expansions for the recurrence coefficients of certain Freud polynomials. Consider the polynomials $p_{n}$ orthonormal on the real line with respect to the weight function

$$
w(x)=\exp (-Q(x))
$$

where $Q(x)$ is a polynomial of even degree:

$$
Q(x)=\sum_{i=0}^{2 m} \alpha_{i} x^{i} \quad\left(m>0 \text { integer, } \alpha_{2 m}>0\right) .
$$

That is, the polynomials

$$
P_{n}(x)=\gamma_{n} x^{n}+\cdots \quad\left(\gamma_{n}>0\right)
$$

are such that

$$
\int_{-\infty}^{\infty} p_{l}(x) p_{n}(x) w(x) d x=\delta_{l n} \quad(l, n \geq 0),
$$

where $\delta_{l n}=1$ if $l=n$ and $\delta_{l n}=0$ otherwise. These polynomials satisfy the recurrence equation

$$
x p_{n}(x)=a_{n+1} p_{n+1}(x)+b_{n} p_{n}(x)+a_{n} p_{n-1}(x) \quad(n \geq 0),
$$

where $a_{n}=\gamma_{n-1} / \gamma_{n}(n \geq 1)$ and $a_{0}=p_{-1}(x)=0$ (see e.g. [2, formula (I.2.4) on p. 17] or [13, formulas (3.2.1) and (3.2.2) on p. 42]). Al. Magnus [8, Theorem 6.1] proved the following about the asymptotic behavior of the coefficients $a_{n}$ and $b_{n}$ :

$$
\lim _{n \rightarrow \infty} a_{n} n^{-1 /(2 m)}=C\left(m, \alpha_{2 m}\right) \stackrel{\operatorname{def}}{=}\left(\alpha_{2 m}\left(\begin{array}{c}
2 m-1 \\
m-1
\end{array}\right)\right)^{-1 /(2 m)}
$$

and

$$
\lim _{n \rightarrow \infty} b_{n} n^{-1 /(2 m)}=0 .
$$

In an earlier paper [7], Magnus established (4.3) in the special case $Q(x)=x^{2 m}$, settling a conjecture of G. Freud [4, p. 5]; see [11, Sections $4.15-4.18$ ] for a discussion of results and conjectures concerning Freud 
weights. D. Lubinsky, H. N. Mhaskar, and E. B. Saff $[6,6 a]$ recently proved the analogue of (4.3) under more general circumstances: e.g. their result applies to the weight function $w(x)=\exp \left(-|x|^{\lambda}\right)$ with $\lambda>1$.

In establishing his result, Magnus considered the Jacobi matrix formed by the coefficients in (4.2), which is the infinite matrix

$$
A=\left[\begin{array}{ccccc}
b_{0} & a_{1} & 0 & 0 & \ldots \\
a_{1} & b_{1} & a_{2} & 0 & \ldots \\
0 & a_{2} & b_{2} & a_{3} & \ldots \\
0 & 0 & a_{3} & b_{3} & \ldots \\
\ldots & \ldots & \ldots & \ldots
\end{array}\right]
$$

He showed that the sequences $a=\left(a_{1}, a_{2}, a_{3}, \cdots\right)$ and $b=$ $\left(b_{0}, b_{1}, b_{2}, \cdots\right)$ satisfy the equations

$$
F_{n}(a, b) \stackrel{\text { def }}{=} a_{n}\left(Q^{\prime}(A)\right)_{n, n-1}=n \quad(n=1,2,3, \ldots)
$$

and

$$
G_{n}(a, b) \stackrel{\text { def }}{=}\left(Q^{\prime}(A)\right)_{n, n}=0 \quad(n=0,1,2, \ldots),
$$

where $Q^{\prime}$ is the derivative of the polynomial in (4.1), and $(B)_{i j}$ denotes the element in the $(i+1)$ st row and $(j+1)$ st column of the matrix $B$. These equations have their origin in earlier works of Freud [3, Lemma 1 on p. 93] and Shohat [12]. Using equations (4.6) and (4.7), we will show by Theorem 1.1 that $a_{n} n^{-1 /(2 m)}$ and $b_{n} n^{-1 /(2 m)}$ have asymptotic expansions in powers of $n^{-1 / m}$. As the first step, we will show that such asymptotic expansions exist in terms of powers of $n^{-1 /(2 m)}$ :

LEMMA 4.1. There are real numbers $c_{\nu l}(\nu+1,2, l=1,2,3, \ldots)$ such that for any integer $N \geq 1$ we have

$$
a_{n} n^{-1 /(2 m)}=C\left(m, \alpha_{2 m}\right)+\sum_{l=1}^{N} c_{1 l^{n}} n^{-l /(2 m)}+o\left(n^{-N /(2 m)}\right)
$$

(see (4.3) for $\left.C\left(m, \alpha_{2 m}\right)\right)$ and

$$
b_{n} n^{-1 /(2 m)}=\sum_{l=1}^{N} c_{2 l} n^{-n /(2 m)}+o\left(n^{-N(2 m)}\right),
$$

as $n \rightarrow \infty$. Here

$$
c_{21}=-\alpha_{2 m-1} /\left(2 m \alpha_{2 m}\right) .
$$

Later we will need the equation

$$
b_{n}=-\alpha_{2 m-1} /\left(2 m \alpha_{2 m}\right)+O\left(n^{-1 /(2 m)}\right),
$$


which is a direct consequence of (4.9) with $N=1$ and (4.10). Magnus stated this equation with the sharper error term $O\left(n^{-1 / m}\right)$ in a preliminary version of his paper [8], but he omitted it from the final version. Our formula (5.2) below implies (4.11) with this sharper error term.

Proof. We are going to take a closer look at equations (4.6) and (4.7). We of course have

$$
Q^{\prime}(A)=\sum_{s=0}^{2 m-1}(s+1) \alpha_{s+1} A^{s},
$$

and so it is of crucial importance to obtain a closer description of the expressions $\left(A^{s}\right)_{n, n}$ and $\left(A^{s}\right)_{n, n-1}$. Notice that

$$
A=\left[a_{i j}\right]_{0 \leq i, j<\infty}
$$

with $a_{i i}=b_{i}, a_{i, i+1}=a_{i+1, i}=a_{i+1}$ and $a_{i j}=0$ if $|i-j|>1$ (cf. (4.5)). Thus

$$
\left(A^{s}\right)_{p q}=\sum_{i_{1}, i_{2}, \ldots, i_{s}} a_{p i_{1}} a_{i_{1} i_{2}} \cdots a_{i_{s} q} \quad(p, q \geq 0),
$$

where $i_{1}, i_{2}, \ldots, i_{s}$ independently run over the values $0,1,2, \ldots$, but if one only wants to consider the nonzero terms in this product, then they are subject to the additional conditions $\left|i_{l}-i_{l+1}\right| \leq 1$ for $0 \leq l \leq s$, where we put $i_{0}=p$ and $i_{s+1}=q$. Thus there are homogeneous polynomials

$$
P_{\mu s}\left(x_{1 j}, x_{2 j}:|j| \leq s / 2\right) \quad(\mu=1,2)
$$

of degree $s$ such that

$$
\left(A^{s}\right)_{n, n-2+\mu}=P_{\mu s}\left(a_{n+j}, b_{n+j}:|j| \leq s / 2\right)
$$

holds for $n>s / 2$. Indeed, the condition $\left|i_{l}-i_{l+1}\right| \leq 1$ on the indices $i_{l}$ in (4.13) imply that

$$
n-(s+1) / 2 \leq i_{l} \leq n+s / 2
$$

in case $p=n$ and $q=n$ or $n-1$, and equality can happen only if $i_{l^{\prime}} \neq i_{l^{\prime}+1}$ for all $l^{\prime}$ with $0 \leq l^{\prime} \leq s$; thus $\left(A^{s}\right)_{n, n-1}$ or $\left(A^{s}\right)_{n, n}$ does not depend on $a_{n+j}$ or $b_{n+j}$ for $j$ outside the range indicated (in fact, the exact range is of no importance). (4.14) is not valid for $n \leq s / 2$, because $a_{i}$ and $b_{j}$ are undefined for $i \leq 0$ and $j<0$. An important observation is that for odd $s$ every term of $P_{2 s}\left(x_{\nu j}\right)$ contains $x_{2 j}$ for some $j$ as a factor. In other words, in (4.13) with $p=q=n>s / 2$ we have $i_{l}=i_{l+1}$ for some $l$ with $0 \leq l \leq s\left(i_{0}=i_{s+1}=n\right)$ in case $s$ is odd. This is because the parity of $0=i_{s}-i_{0}$ is the same as that of

$$
\sum_{l=0}^{s}\left|i_{l+1}-i_{l}\right|
$$


An important consequence of this is that

$$
\frac{\partial}{\partial x_{1 i}} P_{2 s}\left(x_{\nu j}: \nu=1,2,|j| \leq s / 2\right)=0 \quad(|i| \leq s / 2)
$$

holds for odd $s$ at every point with $x_{2 j}=0$ for all $j$ with $|j| \leq s / 2$.

Introduce the variables

$$
\begin{aligned}
& y_{1 n}=a_{n} n^{-1 /(2 m)}, \\
& y_{2 n}=b_{n} n^{-1 /(2 m)},
\end{aligned}
$$

and

$$
\xi_{n}=n^{-1 /(2 m)} .
$$

Dividing both sides of (4.6) by $n$ and taking (4.12) and (4.14) into account, we obtain

$$
H_{\mu}\left(\xi_{n} ; y_{\nu, n+j}: \nu=1,2,|j|<m\right)=0 \quad(\mu=1,2)
$$

for $\mu=1(n \geq m)$ with

$$
\text { (4.20) } \quad \begin{aligned}
H_{1}\left(\xi ; x_{\nu j}: \nu=1,2,|j|<m\right) \\
=-1+x_{10} \sum_{s=0}^{2 m-1}(s+1) \alpha_{s+1} \xi^{2 m-1-s} \\
\cdot P_{1 s}\left(x_{\nu j}\left(1+j \xi^{2 m}\right)^{1 /(2 m)}: \nu=1,2,|j| \leq s / 2\right) .
\end{aligned}
$$

The argument of $P_{1 s}$ is explained by the equation

$$
a_{n+j} n^{-1 /(2 m)}=y_{1, n+j}\left(1+j \xi_{n}\right)^{1 /(2 m)},
$$

and a similar equation involving $b_{n+j}$. The range $|j|<m$ on the left-hand side of (4.20) (and in (4.19), for $\mu=1$ ) is explained by the observation that in each term of the sum in (4.20) we have $|j| \leq s / 2$, and the maximum value for $s / 2$ in (4.20) is $m-1 / 2$. A similar remark applies to (4.21) below (concerning the case $\mu=2$ in (4.19)). Similarly, dividing both sides of (4.7) by $n^{1-1 /(2 m)}$, we obtain (4.19) with $\mu=2$, where

$$
\begin{aligned}
H_{2}(\xi ; & \left.x_{\nu j}: \nu=1,2,|j|<m\right) \\
= & \sum_{s=0}^{2 m-1}(s+1) \alpha_{s+1} \xi^{2 m-1-s} \\
& \cdot P_{2 s}\left(x_{\nu j}\left(1+j \xi^{2 m}\right)^{1 /(2 m)}: \nu=1,2,|j| \leq s / 2\right) .
\end{aligned}
$$


We will use Theorem 1.1 to derive (4.8) and (4.9) from (4.19) at the point

$$
p=\left(0 ; x_{\nu j}: \nu=1,2,|j|<m\right)
$$

with $x_{1 j}=C\left(m, \alpha_{2 m}\right)$ (cf. (4.3)) and $x_{2 j}=0$ for $|j|<m$ replacing the point 0 . Note that the (appropriate modification of) (1.2) is satisfied in view of (4.3) and (4.4). Moreover, $H_{\mu}(\mu=1,2)$ is differentiable infinitely many times in a neighborhood of $p$. As $1 / \beta$ is an integer with $\beta=$ $-1 /(2 m)$, we will get the asymptotic expansion in the simplified form (1.6) rather than (1.5). The only thing that we have to establish is that condition (1.1) with $p$ replacing 0 is satisfied.

Noting that at $p$ we have $\xi=0$, equations (4.20) and (4.21) simplify to

$$
H_{1}\left(0 ; x_{v j}\right)=-1+2 m \alpha_{2 m} x_{10} P_{1,2 m-1}\left(x_{v j}\right)
$$

and

$$
H_{2}\left(0 ; x_{v j}\right)=2 m \alpha_{2 m} P_{2,2 m-1}\left(x_{\nu j}\right),
$$

where $\nu=1,2$ and $|j|<m$. Writing $\left[d_{p q}(z)\right]_{p, q=1,2}$ for the determinant corresponding to the determinant in (1.1), we therefore have

$$
d_{1 q}(z)=\left.\sum_{l=-m+1}^{m-1} z^{l} \frac{\partial}{\partial x_{q l}}\left(x_{10} P_{1,2 m-1}\left(x_{\nu j}\right)\right)\right|_{p}
$$

and

$$
d_{2 q}(z)=\left.\sum_{l=-m+1}^{m-1} z^{l} \frac{\partial}{\partial x_{q l}} P_{2,2 m-1}\left(x_{\nu j}\right)\right|_{p},
$$

where the symbol $\left.\right|_{p}$ indicates that the derivatives have to be taken at the point $\left(x_{0} ; x_{\nu j}\right)=p$. We also dropped the factor $2 m \alpha_{2 m} z^{m-1}$ from each element of the determinant, since this does not affect the validity of (1.1). It is more convenient to have $z^{l}$ in (4.25) and (4.26) than $z^{m-1+l}$; the reason we would get the latter is that here $n-m+1$ corresponds to $n$ in Theorem 1.1. (Thus we get expansion (1.6) in terms of $(n-m+1)^{\beta l}$ rather than $n^{\beta l}$, but using the binomial expansion we can rewrite this in terms of $n^{\beta l}$, as we did it in (3.5).)

Observe that

$$
d_{21}(z)=0
$$

in view of (4.15). Thus to show that the analogue of (1.1) is satisfied we have to show that

$$
d_{p p}(z) \neq 0 \text { whenever }|z|=1 \quad(p=1,2) .
$$


To this end, we will use some results of Magnus [8] concerning the infinite matrix

$$
\begin{aligned}
& {\left[\begin{array}{ll}
\left(a_{k} / 2\right) \partial F_{n}(a, b) / \partial a_{k} & \partial F_{n}(a, b) / \partial b_{k} \\
\left(a_{k} / 2\right) \partial G_{n}(a, b) / \partial a_{k} & \partial G_{n}(a, b) / \partial b_{k}
\end{array}\right] \quad \begin{array}{l}
n=1,2, \ldots \\
n=0,1, \ldots,
\end{array}} \\
& k=1,2, \ldots \quad k=0,1, \ldots
\end{aligned}
$$

where $F_{n}$ and $G_{n}$ are defined by (4.6) and (4.7). According to Theorems 5.2 and 5.3, respectively, of [8], this matrix is symmetric and positive definite for the choice $Q(x)=x^{2 m}, a_{n}=C>0(n \geq 1)$ and $b_{n}=0$ $(n \geq 0)$. This implies that, for the same choice of $a, b$ and $Q$, the matrices

$$
\left[\left(a_{k} / 2\right) \partial F_{n}(a, b) / \partial a_{k}\right]_{k, n=1,2, \ldots}
$$

and

$$
\left[\partial G_{n}(a, b) / \partial b_{k}\right]_{k, n=0,1, \ldots}
$$

are also symmetric and positive definite. In view of (4.12) and (4.14), we have

$$
\frac{\partial F_{n}(a, b)}{\partial a_{k}}=\left.2 m \frac{\partial}{\partial x_{1, n-k}}\left(x_{10} P_{1,2 m-1}\left(x_{\nu j}\right)\right)\right|_{x_{1 J}=C, x_{2 j}=0}
$$

for $n \geq m$, with the same choice of $a, b$ and $Q$ as above (for $n-k \geq m$ the right-hand side is to be interpreted as 0 ). Thus, taking (4.22) into account, the positive definiteness of the matrix in (4.29) with $C=$ $C\left(m, \alpha_{2 m}\right)($ cf. (4.3)) means that

$$
\sum_{n=m}^{\infty} \sum_{k=m}^{\infty} \frac{\partial H_{1}(p)}{\partial x_{1, n-k}} \sigma_{n} \bar{\sigma}_{k} \geq K \sum_{n=m}^{\infty}\left|\sigma_{n}\right|^{2}
$$

holds with some $K>0$ for any sequence $\left(\sigma_{m}, \sigma_{m+1}, \ldots\right)$ of complex numbers such that only finitely many of the $\sigma_{n}$ 's are nonzero ( $\bar{w}$ denotes the complex conjugate of $w$; for $|n-k| \geq m$ the above derivatives are to be interpreted as zero). Taking $\sigma_{n}=z^{n} / \sqrt{M}$ for $m \leq n \leq M$ and $\sigma_{n}=0$ for $n>M$, where $z$ is an arbitrary complex number with $|z|=1$, and making $M \rightarrow \infty, d_{11}(z) \neq 0$ follows from the above inequality. $d_{22}(z) \neq 0$ follows in a similar way from the positive definiteness of the matrix in (4.30). This shows that (4.28) is indeed valid, and so (4.8) and (4.9) follows.

We have yet to establish (4.10). To this end, note that, according to (4.2), the transformation $x^{\prime}=x+\eta$ causes the change $b_{n}^{\prime}=b_{n}+\eta$. Thus, by completing the $m$ th power in (4.1) via the transformation $x \leftarrow x+$ $\alpha_{2 m-1} /\left(2 m \alpha_{2 m}\right)$, we may assume that $\alpha_{2 m-1}=0$; in this case (4.10) 
simplifies to

$$
c_{21}=0 \text {. }
$$

To show this, substitute the estimates

$$
\begin{aligned}
& y_{1 n}=C\left(m, \alpha_{2 m}\right)+O\left(n^{-1 /(2 m)}\right) \text { and } \\
& y_{2 n}=c_{21} n^{-1 /(2 m)}+O\left(n^{-1 / m}\right),
\end{aligned}
$$

valid as $n \rightarrow \infty$ according to (4.8) and (4.9) (cf. (4.16) and (4.17)), into (4.21). In doing so, observe that in view of the assumption $\alpha_{2 m-1}=0$, the contribution of the terms for $0 \leq s \leq 2 m-2$ on the right-hand side is $O\left(\xi_{n}^{2}\right)=O\left(n^{-1 / m}\right)$ (cf. (4.18)). Observe, further, that every term in $P_{2,2 m-1}\left(x_{\nu j}\right)$ has positive coefficient (cf. (4.13) and (4.14)). As we remarked after (4.14), every term of $P_{2,2 m-1}\left(x_{\nu j}\right)$ contains $x_{2 j}$ for some $j$ as a factor. Moreover, by using arguments similar to those described after (4.14), it is easy to see that there are terms of $P_{2,2 m-1}\left(x_{\nu j}\right)$ in which $x_{2 j}$ occurs for exactly one $j$, and this $x_{2 j}$ occurs with exponent 1 . Thus the above substitution gives

$$
H_{2}\left(\xi_{n}, y_{\nu, n+j}\right)=K c_{21} n^{-1 /(2 m)}+O\left(n^{-1 / m}\right)
$$

with a positive constant $K$. Substituting this into (4.19) with $\mu=2$, $c_{21}=0$ follows, verifying (4.31). The proof of the lemma is complete.

5. Improved asymptotic expansions for the Freud coefficients. In (4.8) and (4.9) every second coefficient is zero, that is we can obtain asymptotic expansions in terms of powers of $n^{-1 / m}$. More precisely, Lemma 4.1 can be strengthened as

THEOREM 5.1. For the recurrence coefficients $a_{n}, b_{n}$ of the orthonormal polynomials associated with the weight function $w(x)=\exp (-Q(x))$ with $Q(x)$ as in (4.1), there are real numbers $\eta_{\nu l}(\nu=1,2, l=1,2,3, \ldots)$ such that

$$
a_{n} n^{-1 /(2 m)}=C\left(m, \alpha_{2 m}\right)+\sum_{l=1}^{N} \eta_{1 l^{n^{-l / m}}+o\left(n^{-N / m}\right)}
$$

and

$$
b_{n}=-\alpha_{2 m-1} /\left(2 m \alpha_{2 m}\right)+\sum_{l=1}^{N} \eta_{2 l} n^{-l / m}+o\left(n^{-N / m}\right)
$$

hold for any integer $N \geq 1$ as $n \rightarrow \infty$, where $C\left(m, \alpha_{2 m}\right)$ is given by (4.3). 
The particular case $m=2$ of this result is briefly discussed in [1]. Extending an earlier result of J. S. Lew and D. A. Quarles Jr. [5] for $Q(x)=x^{4}$, Máté-Nevai-Zaslavsky [10, Theorem 1 on $\mathrm{p}$. 497] established a stronger version of (5.1) for $Q(x)=x^{2 m}$, with the asymptotic expansion on the right-hand side being given in terms of powers of $n^{-2}$.

Proof. Similarly as at the end of the proof of Lemma 4.1, while establishing (4.10), we may assume that $\alpha_{2 m-1}=0$. Then, according to (4.11) we have

$$
b_{n}=O\left(n^{-1 /(2 m)}\right) \text {. }
$$

Instead of (4.16) and (4.17), we now introduce the notation

$$
y_{1 n}=a_{n} n^{-1 /(2 m)} \text { and } y_{2 n}=b_{n} \text {. }
$$

Then we still have

$$
\lim _{n \rightarrow \infty} y_{1 n}=C\left(m, \alpha_{2 m}\right)
$$

and

$$
\lim _{n \rightarrow \infty} y_{2 n}=0
$$

according to (4.3) and (5.3), so it is still the point $p$ described in (4.22) that replaces 0 in the application of Theorem 1.1.

We now put

$$
\xi_{n}=n^{-1 / m}
$$

instead of (4.18), and we will obtain the analogue

$$
H_{\mu}^{*}\left(\xi_{n} ; y_{\nu, n+j}: \nu=1,2,|j|<m\right)=0 \quad(\mu=1,2)
$$

of (4.19) via dividing (4.6) by $n$ and (4.7) by $n^{1-1 / m}$. As (5.4) and (5.5) imply $a_{n}=y_{1 n} \xi_{n}^{-1 / 2}$, according to (4.12) and (4.14) we have

$$
\begin{aligned}
H_{1}^{*}\left(\xi ; x_{\nu j}: \nu=1,2,|j|<m\right) \\
=-1+\xi^{m}\left(x_{10} \xi^{-1 / 2}\right) \sum_{s=0}^{2 m-1}(s+1) \alpha_{s+1} \\
\quad \cdot P_{1 s}\left(x_{1 j} \xi^{-1 / 2}\left(1+j \xi^{m}\right)^{1 /(2 m)}, x_{2 j}:|j|<s / 2\right) .
\end{aligned}
$$

Here $\xi^{m}$ appears in front of the second term on the right because $\xi_{n}^{m}=1 / n$, and we divided (4.6) by $n$. Similarly,

$$
\begin{aligned}
H_{2}^{*}( & \left.\xi ; x_{\nu j}: \nu=1,2,|j|<m\right) \\
= & \xi^{m-1} \sum_{s=0}^{2 m-1}(s+1) \alpha_{s+1} \\
& \cdot P_{2 s}\left(x_{1 j} \xi^{-1 / 2}\left(1+j \xi^{m}\right)^{1 /(2 m)}, x_{2 j}:|j|<s / 2\right) .
\end{aligned}
$$


Observe that $H_{\mu}^{*}$ is differentiable in a neighborhood of $p$ (cf. (4.22)). Indeed, it is easy to see that, on the one hand, the positive powers of $\xi$ in (5.7) and (5.8) at least cancel out the negative powers, and so $\xi$ does not occur with negative exponent in $H_{\mu}^{*}$. On the other hand, $\xi^{1 / 2}$, which is not differentiable at 0 , does not occur in $H_{\mu}^{*}$. This is so because in every term of (4.6) and (4.7) each $a_{k}$ occurs with an even exponent. In fact, in each term on the right-hand side of (4.13), for $p=q=n$ each factor $a_{k-1, k}$ has to be matched by a corresponding factor $a_{k, k-1}$, so $a_{k}$ occurs with even power in each term of $\left(A^{s}\right)_{n, n}$. The same is true for $p=n$ and $q=n-1$ except for $k=n$ : there has to be an extra occurrence of $a_{n, n-1}$, not matched by $a_{n-1, n}$. Thus in $\left(A^{s}\right)_{n, n-1}$ each $a_{k}$ occurs with an even power except for $a_{n}$, which always occurs with an odd power; but the extra factor $a_{n}$ occurring (4.6) compensates for this. Thus, indeed, in every term of (4.6) and (4.7) each $a_{k}$ occurs with an even exponent. In other words, in every term of (5.7) and (5.8), each expression $x_{1 j} \xi^{-1 / 2}$ occurs with an even exponent; thus $\xi^{1 / 2}$ indeed does not occur in $H_{\mu}^{*}$.

Hence, to be able to apply Theorem 1.1 we have only to show that the analogue of (1.1) is satisfied for $H_{\mu}^{*}$ at $p$. We will show this by verifying the equations

$$
\frac{\partial H_{\mu}^{*}(p)}{\partial x_{1 j}}=\frac{\partial H_{\mu}(p)}{\partial x_{1 j}} \quad(\mu=1,2)
$$

and

$$
\frac{\partial H_{2}^{*}(p)}{\partial x_{2 j}}=\frac{\partial H_{2}(p)}{\partial x_{2 j}},
$$

where $H_{\mu}$ are as in (4.20) and (4.21) and $|j|<m$. This will clearly suffice in view of (4.27) and (4.28) (cf. (4.25) and (4.26)). If we substitute $\xi=0$ into (5.7) and (5.8), then, similarly to (4.23) and (4.24), we obtain

$$
H_{1}^{*}\left(0 ; x_{\nu j}\right)=-1+2 m \alpha_{2 m} x_{10} P_{1,2 m-1}^{*}\left(x_{\nu j}\right)
$$

and

$$
H_{2}^{*}\left(0 ; x_{\nu j}\right)=2 m \alpha_{2 m} P_{2,2 m-1}^{*}\left(x_{\nu j}\right) \text {. }
$$

Here the polynomial $P_{1,2 m-1}^{*}\left(x_{\nu j}\right)$ is formed as the sum of those terms of $P_{1,2 m-1}\left(x_{\nu j}\right)$ which do not contain any $x_{2 j}$. Indeed, as $P_{1,2 m-1}\left(x_{\nu j}\right)$ is a homogeneous polynomial of degree $2 m-1$, for those of its terms $T\left(x_{\nu j}\right)$ containing an $x_{2 j}$, the degree of $T\left(x_{\nu j}\right)$ in the $x_{1 j}$ 's is $<2 m-1$. Thus the expression

$$
\xi^{m}\left(x_{10} \xi^{-1 / 2}\right) T\left(x_{1 j} \xi^{-1 / 2}\left(1+j \xi^{m}\right)^{1 /(2 m)}, x_{2 j}\right)
$$


will contain $\xi$ with a positive exponent. Therefore the substitution $\xi=0$ will cancel this term on the right-hand side of (5.7). Similarly, $P_{2,2 m-1}^{*}\left(x_{\nu j}\right)$ is formed as the sum of those terms of $P_{2,2 m-1}\left(x_{\nu J}\right)$ which contain only one $x_{2 j}$, and that with exponent 1 . It is important to recall here that, as remarked after (4.14), every term of $P_{2,2 m-1}\left(x_{\nu j}\right)$ contains at least one $x_{2 j}$. Observe, furthermore, that the term corresponding to $s=2 m-2$ in the sum on the right-hand side of $(5.8)$ would also contribute to the right-hand side of (5.12) expressing $H_{2}^{*}\left(0, x_{\nu j}\right)$ except for the fact that we assumed $\alpha_{2 m-1}=0$ above, at the beginning of this proof.

Now, as we have $x_{2 j}=0$ for every $j(|j|<m)$ at the point $p$, it is clear from the above description of the polynomials $P_{\mu, 2 m-1}^{*}$ that

$$
\begin{aligned}
& P_{\mu, 2 m-1}^{*}(p)=P_{\mu, 2 m-1}(p) \quad(\mu=1,2), \\
& \frac{\partial P_{\mu, 2 m-1}^{*}(p)}{\partial x_{1 j}}=\frac{\partial P_{\mu, 2 m-1}(p)}{\partial x_{1 j}} \quad(\mu=1,2), \quad \text { and } \\
& \frac{\partial P_{2,2 m-1}^{*}(p)}{\partial x_{2 j}}=\frac{\partial P_{2,2 m-1}(p)}{\partial x_{2 j}}
\end{aligned}
$$

hold $(|j|<m)$. In view of these equations, (5.9) and (5.10) follow by comparing (5.11) and (5.12) to (4.23) and (4.24), respectively. This completes the proof of the theorem.

\section{REFERENCES}

[1] William C. Bauldry, Attila Máté, and Paul Nevai, Asymptotic expansions of recurrence coefficients of asymmetric Freud polynomials, in Approximation Theory V, Proceedings of the 1986 Conference on Approximation Theory at Texas A \& M University, Academic Press, 1986, pp. 251-254.

[2] G. Freud, Orthogonal Polynomials, Pergamon Press, Oxford and New York, 1966.

[3] _ On the greatest zero of an orthogonal polynomial, Acta. Sci. Math., (Szeged) 34 (1973), 91-97.

[4] - On the coefficients in the recursion formulae of orthogonal polynomials, Proc. Roy. Irish Acad., Sect. A, 76 (1976), 1-6.

[5] J. S. Lew and D. A. Quarles, Jr., Nonnegative solutions of a nonlinear recurrence, J. Approx. Theory, 38 (1983), 357-379.

[6] D. S. Lubinsky, H. N. Mhaskar, and E. B. Saff, Freud's conjecture for exponential weights, Bull. Amer. Math. Soc., 15 (1986), 217-221.

[6a] _ A proof of Freud's conjecture for exponential weights, Constructive Approximation, to appear.

[7] Al. Magnus, A proof of Freud's conjecture about the orthogonal polynomials related to $|x|^{\rho} \exp \left(-x^{2 m}\right)$ for integer $m$, in: Polynômes Orthogonaux et Applications, Proceedings of the Laguerre Symposium held at Bar-le-Duc, C. Brézinski, A. Draux, A. P. Magnus, P. Maroni, A. Ronvaux, ed., Lecture Notes in Math. 1171, Springer-Verlag, New York, 1985; pp. 362-372. 

65-99.

On Freud's equations for exponential weights, J. Approx. Theory, 46 (1986),

[9] A. Máté and P. Nevai, Asymptotics for solutions of smooth recurrence equations, Proc. Amer. Math. Soc., 93 (1985), 423-429.

[10] A. Máté, P. Nevai, and T. Zaslavsky, Asymptotic expansions of ratios of coefficients of orthogonal polynomials with exponential weights, Trans. Amer. Math. Soc., 287 (1985), 495-505.

[11] P. Nevai, Géza Freud, Christoffel functions and orthogonal polynomials, J. Approx. Theory, 48 (1986), 3-167.

[12] J. Shohat, A differential equation for orthogonal polynomials, Duke Math. J., 5 (1939), 401-417.

[13] G. Szegö, Orthogonal polynomials, 4th ed., Amer. Math. Soc. Colloq. Publ. Vol. 23, Amer. Math. Soc., Providence, RI, 1978.

Received June 16, 1986. This material is based upon work supported by the Graduate Student Alumni Research Award Program under Grant No. 0349 (first author), by the National Science Foundation under Grant Nos. DMS-8400906 (second author) and DMS-8419525 (third author), by the PSC-CUNY Research Award Program of the City University of New York under Grant No. 6-65263 (second author), by the Fulbright Grant Program of the U. S. Information Agency under Grant No. 85-41612 (third author), and by the Universite Catholique de Louvain in Belgium (third author). The second author made his contribution as a member of the Institure for Advanced Study on an RCA Fellowship during the 1985-86 academic year, and he wishes to thank the RCA Corporation for its support.

ApPalachian State UnIVERSITY

BOON, NC 28608

OHIo State UnIVERSITY

Columbus, OH 43210 (first author)

BROOKLYN COLLEGE

OF THE City UNIVERSITY OF NeW YORK

BROOKLYN, NY 11210

INSTITUTE FOR ADVANCED STUDY

PRINCETON, NJ 08543 (second author)

AND

UNIVERSITY OF SOUTH CAROLINA

Columbia, SC 29208

OHIo State University

Columbus, OH 43210 (third author) 



\title{
PACIFIC JOURNAL OF MATHEMATICS
}

\section{EDITORS}

\author{
V. S. VARADARAJAN \\ (Managing Editor) \\ University of California \\ Los Angeles, CA 90024 \\ HERBERT ClEMENS \\ University of Utah \\ Salt Lake City, UT 84112 \\ R. FINN \\ Stanford University \\ Stanford, CA 94305
}

\author{
HERMANN FLASCHKA \\ University of Arizona \\ Tucson, AZ 85721
}

RAMESh A. GANGOLLI University of Washington Seattle, WA 98195

VAUGHAN F. R. JONES University of California

Berkeley, CA 94720

\author{
ROBION KIRBY \\ University of California \\ Berkeley, CA 94720 \\ C. C. MOORE \\ University of California \\ Berkeley, CA 94720 \\ HAROLD STARK \\ University of California, San Diego \\ La Jolla, CA 92093
}

\section{ASSOCIATE EDITORS}

\author{
R. ARENS \\ E. F. BECKENBACH \\ B. H. NEUMANN \\ F. WOLF \\ K. YOSHIDA \\ (1906-1982)

\section{SUPPORTING INSTITUTIONS}

\begin{abstract}
UNIVERSITY OF ARIZONA
UNIVERSITY OF BRITISH COLUMBIA

UNIVERSITY OF CALIFORNIA

MONTANA STATE UNIVERSITY

UNIVERSITY OF NEVADA, RENO

NEW MEXICO STATE UNIVERSITY

OREGON STATE UNIVERSITY
\end{abstract} \\ CALIFORNIA INSTITUTE OF TECHNOLOGY \\ UNIVERSITY OF OREGON \\ UNIVERSITY OF SOUTHERN CALIFORNIA \\ STANFORD UNIVERSITY \\ UNIVERSITY OF HAWAII \\ UNIVERSITY OF TOKYO \\ UNIVERSITY OF UTAH \\ WASHINGTON STATE UNIVERSITY \\ UNIVERSITY OF WASHINGTON
}

The Supporting Institutions listed above contribute to the cost of publication of this Journal, but they are not owners or publishers and have no responsibility for its content or policies.

Mathematical papers intended for publication in the Pacific Journal of Mathematics should be in typed form or offset-reproduced (not dittoed), double spaced with large margins. Please do not use built up fractions in the text of the manuscript. However, you may use them in the displayed equations. Underline Greek letters in red, German in green, and script in blue. The first paragraph must be capable of being used separately as a synopsis of the entire paper. In particular it should contain no bibliographic references. Please propose a heading for the odd numbered pages of less than 35 characters. Manuscripts, in triplicate, may be sent to any one of the editors. Please classify according to the scheme of Math. Reviews, Index to Vol. 39. Supply name and address of author to whom proofs should be sent. All other communications should be addressed to the managing editor, or Elaine Barth, University of California, Los Angeles, California 90024.

There are page-charges associated with articles appearing in the Pacific Journal of Mathematics. These charges are expected to be paid by the author's University, Government Agency or Company. If the author or authors do not have access to such Institutional support these charges are waived. Single authors will receive 50 free reprints; joint authors will receive a total of 100 free reprints. Additional copies may be obtained at cost in multiples of 50 .

The Pacific Journal of Mathematics is issued monthly as of January 1966. Regular subscription rate: $\$ 190.00$ a year (5 Vols., 10 issues). Special rate: $\$ 95.00$ a year to individual members of supporting institutions.

Subscriptions, orders for numbers issued in the last three calendar years, and changes of address should be sent to Pacific Journal of Mathematics, P.O. Box 969, Carmel Valley, CA 93924, U.S.A. Old back numbers obtainable from Kraus Periodicals Co., Route 100, Millwood, NY 10546.

The Pacific Journal of Mathematics at P.O. Box 969, Carmel Valley, CA 93924 (ISSN 0030-8730) publishes 5 volumes per year. Application to mail at Second-class postage rates is pending at Carmel Valley, California, and additional mailing offices. Postmaster: send address changes to Pacific Journal of Mathematics, P.O. Box 969, Carmel Valley, CA 93924.

\section{PUBLISHED BY PACIFIC JOURNAL OF MATHEMATICS, A NON-PROFIT CORPORATION}




\section{Pacific Journal of Mathematics}

\section{Vol. 133, No. 2 \\ April, 1988}

William Charles Bauldry, Attila Mate and Paul Nevai, Asymptotics for solutions of systems of smooth recurrence equations . . . . . . . . . . 209

Ehrhard Behrends, Isomorphic Banach-Stone theorems and isomorphisms which are close to isometries ............................229

Fernanda Maria Botelho, Rotation sets of maps of the annulus .........251

Edward Graham Evans, Jr. and Phillip Alan Griffith, Binomial behavior

of Betti numbers for modules of finite length . ................. 267

Andrei Iordan, Pseudoconvex domains with peak functions at each point of

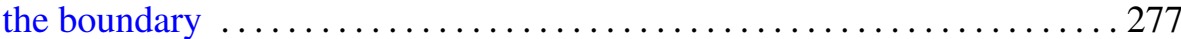

Zyun'iti Iwase, Dehn-surgery along a torus $T^{2}$-knot $\ldots \ldots \ldots \ldots \ldots \ldots . \ldots 289$

Marko Kranjc, Embedding 2-complexes in $\mathbf{R}^{4} \ldots \ldots \ldots \ldots \ldots \ldots \ldots \ldots \ldots$

Aloys Krieg, Eisenstein-series on real, complex, and quaternionic

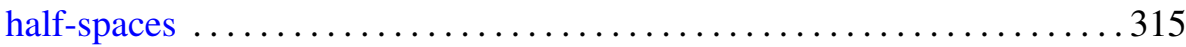

Masato Kuwata, Intersection homology of weighted projective spaces and

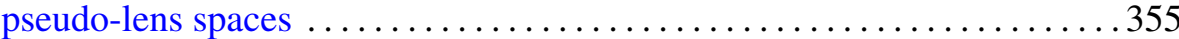

Carl Pomerance, András Sárközy and Cameron Leigh Stewart, On

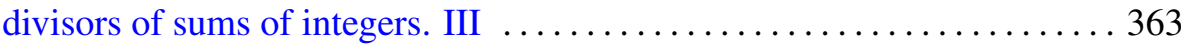

Martin Schechter, Potential estimates in Orlicz spaces ............... 381 